\title{
Steps to the clinic with ELF EMF
}

\author{
Ash Madkan ${ }^{1^{*}}$, Martin Blank ${ }^{2}$, Edward Elson ${ }^{3}$, Kuo-Chen Chou ${ }^{4}$, Matthew S. Geddis ${ }^{5}$, Reba \\ Goodman $^{1}$ \\ ${ }^{1}$ Department of Pathology, Anatomy and Cell Biology, College of Physicians and Surgeons, Columbia University, New York, USA \\ ${ }^{2}$ Department of Physiology and Cellular Biophysics, College of Physicians and Surgeons, Columbia University, New York, USA \\ ${ }^{3}$ Department of Electrical and Computer Engineering, College Park, University of Maryland, MD, USA \\ ${ }^{4}$ Gordon Life Science Institute, San Diego, California, USA \\ ${ }^{5}$ Department of Science, Borough of Manhattan Community College-CUNY, New York, NY
}

Received 10 October 2009; revised 25 October 2009; accepted 27 October 2009.

\begin{abstract}
There have been many models to identify and analyze low-frequency motions in protein and DNA molecules. It has been successfully used to simulate various low-frequency collective motions in protein and DNA molecules. Lowfrequency motions in biomacromolecules originate from two common and intrinsic characteristics; i.e., they contain 1) a series of weak bonds, such as hydrogen bonds, and 2) a substantial mass distributed over the region of these weak bonds. Many biological functions and dynamic mechanisms, including cooperative effects have been reported. In this regard, some phenomenological theories were established. However, differences in experimental outcomes are expected since many factors could influence the outcome of experiments in EMF research. Any effect of EMF has to depend on the energy absorbed by a biological organism and on how the energy is delivered in space and time. Frequency, intensity, exposure duration, and the number of exposure episodes can affect the response, and these factors can interact with each other to produce different effects. In addition, in order to understand the biological consequence of EMF exposure, one must know whether the effect is cumulative, whether compensatory responses result, and when homeostasis will break down. Such findings will have great potential for use in translation medicine at the clinical level without being invasive.
\end{abstract}

Keywords: Electromagnetic Fields; Hsp70; Interaction Mechanisms; Low-Frequency Collective Motion

\section{INTRODUCTION}

Current investigations primarily focus on electromag- netic fields as electropollutants, e.g. cell phones, with slight regard to therapy. Electro-pollutants are manifestly different in field strength and frequency in comparison to therapeutic applications, yet the FDA fails to address differences in design, implementation and field strength, thereby considering them the same and lists therapeutic devices as "potentially dangerous" by association. The World Health Organization convened scientists from around the world and determined that field strengths less than 20,000 Gauss, which is lower in intensity than magnetic resonance imaging, MRI, are free of adverse side effects [1].

Regardless of device design, EMF technology has been shown to be clinically effective in bone healing [2-4], wound repair [2,5] and neural regeneration [5-10]. In terms of clinical application, EMF-induction of elevated levels of hsp70, a stress response protein, also confers protection against hypoxia [11], aids myocardial function and survival [12] as well as survival following ischemia reperfusion [12-14]. Given these results, we are particularly interested in the translational significance of effect vs. efficacy. This relationship is generally not investigated nor reported. More precise description of EM pulse, field strength intensity and sine wave parameters will provide consistency and scientific basis in reporting findings. It is contended that pulsed therapeutic fields are usually more effective if less than 20 Gauss and frequencies are less than $300 \mathrm{~Hz}$, below which they are referred to as extremely low frequency (ELF) [15-18]. Cell phones are several magnitudes of order larger in both considerations. Most therapeutic ELF-EMF used for wound healing and bone repair use field strengths as small as 50 milliGauss. MRI, a diagnostic EM technology, employs static fields from 15,000 to 50,000 Gauss coupled to radiowaves for tissue penetration. In terms of molecular effects, concern might be expressed for repetitive transcranial magnetic stimulation (rTMS), an EM treatment for mental illness employing extremely low frequencies (ELF) combined with field strengths of sev- 
eral thousand Gauss.

This review does not extend to the remainder of the electromagnetic spectrum (radiofrequency, microwave or infrared spectrum) though much work on the employment of these forms of energy in the treatment of neoplastic disease is currently under investigation. This research has now been employed as adjunctive therapy in clinical oncology, e.g. microwave hyperthermia. The radiofrequency and microwave parts of the spectrum are used by inserting bulk energy, whereas the ELF-EMF region is useful by producing electro-mechanical effects on target tissues, producing specific biochemical reactions.

\section{THE PROMISE OF ELF-EMF IN TREATING CANCER}

Since the 1980s a considerable number of reports have appeared [17,19-22] describing a great variety of cell culture systems, animal models (mice for the most part), field sources producing a variety of waveforms and field strengths, and great variation in exposure protocols. Many of the invitro (cell culture) studies of tumor-cell lines report significant cell-killing compared to control cultures. The studies involving transplantation of tumor lines into mice, frequently subcutaneously into the abdomen, report that magnetic field exposures significantly reduce the size of transplanted tumors (i.e. cyto-reduction) compared to controls. Although it is difficult to compare studies quantitatively, it is interesting that cytoreduction occurs across a whole variety of field strengths, wave-forms, pulsed fields versus sinusoidal fields, and exposure durations. The great challenge for investigators will be to find an optimal exposure regime which delivers the most efficient cancer-cell-killing with the most minimal side-effects, to establish the role of ELFEMF, whether as adjunctive or primary therapy, to relate its efficacy to different kinds of cancer and to the stage and grade of human cancer. This process has not actually begun but some information from animal studies is already providing some direction [23-24].

A few studies have been chosen to illuminate the value of the information they are reporting with regard to guidelines for human investigations not yet begun. It should be pointed out that although China and Russia have reported success in ELF-EMF treatments in human subjects with cancer, documentation is often weak and validation by Western science has not occurred. We can crudely separate the studies into those employing "weak" magnetic fields, not exceeding tens of microTesla and those employing "strong" fields, above one milliTesla and ranging through 100 milliTesla and even up to several Tesla. This is indeed crude because other parameters of the fields, such as waveforms, time and spatial rate of variation of the fields are also critically important. For reference, the geomagnetic field is about 50 microTesla. Some house-hold appliances produce up to 1 milliTesla at a distance of $30 \mathrm{~cm}$ from the body, but usually for short periods. A more prolonged exposure is one to an electric blanket $(60 \mathrm{Hertz})$ in which fields at the surface run from 2 to 5 microTesla [25]. These ambient exposures are considered safe.

One study of extremely low frequency (ELF) pulsedgradient magnetic fields inhibited malignant tumor growth through different biological mechanisms [83] using a pulsed-gradient magnetic field (0.6-2.0 Tesla, gradient of 10-100 T/meter, pulse width of 20-200 milliseconds, frequency of $0.16-1.34 \mathrm{~Hz}$ ) to exposed sarcomas inoculated into the legs of mice. These normally rapidly growing tumors showed significant shrinkage with exposure as compared with a control group. Endothelial cells of tumor blood vessels were swollen and appeared occluded and morphologic observation and biochemical tests revealed marked programmed cell death (apoptosis). Necropsy revealed no abnormalities in normal tissues.

Another study [26] exposed mice with implanted murine 16/C mammary adenocarcinoma cells to a rectified, 60 Hertz magnetic field for 10 minutes per day at 10,15 and 20 milliTesla for 12 consecutive days after a seven day period in which the cells produce visible tumors. Exposure to the fields significantly reduced tumor growth. On microscopic examination the tumor exhibited marked necrosis and evidence of inhibition of tumor vascularization. Necropsy revealed no abnormalities in the remainder of normal tissues.

De Seze et.al., [27] used a 0.8 Hertz square wave 100 $\mathrm{mT}, 8$ hours/day or until death on mice subjected to chemically induced tumor using benzo(a)pyrene. A significant decrease in tumor growth and increase in survival were observed.

Cameron, et. al. [28] transplanted a human breast cancer cell line into athymic nude mice and compared the effects of a rectified $60 \mathrm{~Hz}$ magnetic field signal at 15 milliTesla to that of radiation, $200 \mathrm{cGy}$ of radiation every other day and found significant cyto-reduction in radiation and ELF-EMF exposed mice to a roughly comparable extent. Mice that received either therapy also had significantly fewer lung metastatic sites than did untreated mice. Normal tissues were unaffected. This study raised the question of whether ELF-EMF exposures could achieve the same efficacy as radiation but without the side effects of radiation.

Although electromagnetic technology was originally described by Maxwell in 1865, electromagnetic technology as therapy received little interest from basic scientists or clinicians until the 1980s. It now includes applications such as mitigation of inflammation (electrochemistry) and stimulation of several specific of genes $[15,16]$. Studies on DNA have provided an understanding of cell response to low energy EMF inputs via elec- 
tromagnetically responsive elements (EMRE; EMFsensitive base pairs nCTCTn) [19,20,29,30,84].

\section{SELECTING MODEL SYSTEM FOR TRANSLATIONAL RESEARCH AND ITS CLINICAL POTENTIAL}

Of under rated importance in investigating ELF EMF reactions, is the model system on which experiments are based. When extrapolating preclinical testing results to the intended clinical setting, it is important to recognize and appreciate both the relevant attributes and the limitations of a selected animal. It is essential to select a model system that will help provide an understanding of the interaction mechanism and provide specific markers for studying the effect of EMF on many diseases/ conditions including regeneration; ischemia-reperfusion, and tumor suppression. Such model systems include tissue cultured cells, Platyhelminths (worms), yeast, Diptera (flies), bacteria, fish and mice.

\section{CURRENT THEORIES ON ELF-EMF INTERACTION MECHANISM}

We confine the current discussion to studies of frequencies below $0.3 \mathrm{kHz}$ (commonly defined as the upper limit of ELF) to magnetic fields with sinusoidal waveshapes, studies with simultaneous static magnetic fields, and studies using pulsed magnetic fields. In the many studies reported since the 1980s a variety of different exposure protocols, with variation in such parameters as magnetic field strength, frequency, duration of exposure and combined modality applications with chemotherapy and/or radiation, have been reported. Many studies have not been replicated, a general weakness of the field. But the studies taken as a whole still confer great potential promise on the role of magnetic field therapy, either as adjunctive therapy (i.e. in addition to chemotherapy or ionizing radiation) or even potentially as a primary (stand-alone) therapy for certain neoplastic diseases.

\subsection{Basic Mechanisms Underlying the Efficacy of Elf-Emf Treatments.}

Enough is known about the cellular and molecular mechanisms of interactions of ELF-EMF to furnish a rational basis for employing such a therapeutic modality. We describe several mechanisms for which there is some documentation and a few proposed models, not verified, which we believe are worth further investigation.

One strongly documented mechanism is the activation of apoptosis, a process characterized as programmed cell death. In response to many different stimuli, a series of biochemical cascades are activated within a cell which results in its death. From a teleological aspect such a process constitutes a defense of the organism as a whole, as a damaged cell can undergo the unregulated proliferation (lack of apoptosis) we regard as a cancerous transformation. Under the microscope such cells can show fragmentation of nuclei, bizarre appearances of nuclear chromatin, membrane blebs and cell shrinkage. Biochemically one observes the activation of a family of cysteine proteases called caspases which destroy structural elements of the cell [21]. Caspases are activated by the $\mathrm{BCl}-2$ family of proteins which cause the release of cytochrome $\mathrm{c}$ from mitochondria into the cytoplasm by altering the mitochondrial membrane potential. Cytochrome $\mathrm{c}$ is considered one of the major factors in activating the caspase cascade. It has been proposed [4] that ELF-EMF can intervene in this process by affecting voltage-dependent anion channels which are used in the $\mathrm{BCl}-2$ activation process. Other processes have also been described as causing increased intra-cytoplasmic calcium through voltage-activated channels as well [31]. One must bear in mind that the broad picture is far more complex, as there is a related literature showing that ELF-EMF fields also play a role in cell proliferation. Such an effect is generally associated with the weak fields, 10 microTesla to a few hundred microTesla, as opposed to higher strength fields, greater than one milliTesla, which can produce cell injury and apoptosis. It has also been reported that a static field combined with an ELF field increases apoptosis [31].

A second mechanism which has been reported from several studies is an effect of ELF-EMF which produces inhibition of new blood vessel formation. This is most dramatically observed in histological sections of tumors exposed to ELF-EMF. Malignant tumors normally elaborate angiogenic factors which cause neo-vascularization of the growing tumor, a proliferation of thinwalled capillaries branching through the tumor mass to provide nutrients to the tumor cells. The endothelial cells which form buds to new vessels from existing vessels appear to be inhibited during and following ELF-EMF exposure, a process which most likely plays a role in tumor cell death.

A few theoretical models have received much discussion over the years. One is the ion cyclotron resonance (ICR) model of McLeod and Liboff [32]. In this model free ions move in a cell membrane in a combined static and ELF magnetic field of the correct "cyclotron" frequency. This motion is thought to trigger cell signals and disrupt normal cell behavior. Another theory is that of Lednev [33] and describes the interaction of magnetic fields with ions bound to channel proteins which influence the opening and closing of the channels.

A variety of stimulative [4] effects from ELF-EMF have been found, as opposed to strong field effects described as destructive. Utilizing primarily cell culture, 
time-varying magnetic fields, sinusoidal or pulsed, with frequencies in the extremely low frequency (ELF range), or repetition rates on the order of 1 to $10 \mathrm{~Hz}$, have been shown to produce up-regulation of early response genes and stress response genes [4], transcription in several different cell lines [16] induction of stress response genes [34], induction of DNA synthesis in fibroblasts [32] induction of DNA synthesis in frog erythrocytes [35] and alterations in the mitotic cycle of sea urchin embryos [36]. The literature has been reviewed [25].

The model of gene regulation was believed to be that the negatively charged DNA was tightly wrapped up in the nucleus with positively charged histones, and that most genes were 'turned off' most of the time. Of course, different regions of the DNA code re being read more or less all the time to replenish essential proteins.

The ability of relatively weak EMF (in the ELF frequency range) to affect movement of electrons has been demonstrated in several specific biological reactions that are fundamental to cellular mechanisms; $\mathrm{Na}$, K-ATPase reaction, the oxidation of cytochrome oxidase, and the oxidation of malonic acid (the Belousov-Zhabotinsky reaction) reviewed in $[3,4]$. Thus, the same fields can cause electrons to move in DNA, leading to areas of local charging and local deaggregation of DNA strands. This would set in motion the biosynthesis associated with the stresses.

This protective mechanism induces the expression of stress response genes and refolds damaged proteins to transport them across cell membranes. Specific DNA sequences on the promoter of the HSP70 stress gene are responsive to EMF, and studies with model biochemical systems suggest that EMF could interact directly with electrons in DNA. The sensitive base pairs are upstream on the HSP70 promoter and consist of the nCTCTn consensus sequence. When the EMRE are tranfected into a reporter gene, which was previously unresponsive to electromagnetic fields, they become sensitive. Studies have shown that the ERK 1-2 protein is phosphorylted when exposed to electromagnetic radiation $(8 \mathrm{mT})$. A related model is that of Elson [27] which, like the model of Blank and Goodman, describes a direct electro-mechanical interaction of ELF-EMF with DNA. These theories have benefited from the results of a number of studies on the ability of DNA to conduct electric currents along the DNA backbone [38]. The phenomenon of DNA conductivity has been demonstrated in vitro using photochemical techniques and direct measurements of current flow through DNA strands using nano-techniques [39]. The phenomenon has not been demonstrated in vivo, but it has been speculated that the motion of charges in DNA could serve to protect DNA from oxidative damage. The model advocated by Elson suggests that charge motion through helical pathways could also serve to open DNA strands, producing origin sites for DNA replication. This model also indicates how very strong magnetic fields could damage DNA, which would send the signal for the induction of apoptosis. This might constitute yet another mechanism for the treatment of tumors. The model may offer an explanation for the finding of DNA strand breaks produced by electromagnetic fields as reported in a few studies [40-42].

A final mechanism first described by Dr. K. C. Chou and subsequently elaborated by both Dr. Chou and Dr. Glen Gordon [43,44] is the concept of low-frequency phonons (or internal motion) in proteins. Dr. Chou reported this mechanism in order to solve a perplexing "free-energy deficit" problem [45], which was encountered in studying the binding interaction between insulin and the insulin receptor [46]. According to the inference elaborated in [45], the wave numbers of the low-frequency phonons were in the range of $10 \sim 100 \mathrm{~cm}^{-1}$, corresponding to the range of terahertz frequency $\left(3 \times 10^{11}\right.$ to $3 \times 10^{12} \mathrm{~Hz}$ ). In the mean time, the possible biological functions of low-frequency phonons in proteins were also discussed [45].

Subsequently, low-frequency modes have been indeed observed by Raman spectroscopy for a number of protein molecules [47,48] and different types of DNA [49-52]. These observed results have also been further confirmed by the neutron scattering experiments [53].

To identify and analyze this kind of low-frequency motion in protein and DNA molecules, the quasi-continuum model was developed [54-60]. It has been successfully used to simulate various low-frequency collective motions in protein and DNA molecules, such as accordion-like motion, pulsation or breathing motion, as reflected by the fact that the low-frequency wave numbers thus derived were quite close to the experimental observations $[54-56,59,61]$. It was also revealed through the quasi-continuum model that the low-frequency motions in biomacromolecules originate from their two common and intrinsic characteristics; i.e., they usually contain 1) a series of weak bonds, such as hydrogen bonds, and 2) a substantial mass distributed over the region of these weak bonds [62].

The most interesting fact is that many marvelous biological functions and their profound dynamic mechanisms, such as cooperative effects [60,63], allosteric transition $[64,65]$, and intercalation of drugs into DNA $[66,67]$, can be revealed through the low-frequency collective motion or resonance in protein and DNA molecules. In this regard, some phenomenological theories $[57,65,67,68]$ were established. Meanwhile, the solitary wave motion was also used to address the internal motion during microtubule growth [69]. A soliton is a selfreinforcing solitary wave (a wave packet or pulse) that maintains its shape while it travels at constant speed. The relationship between the solitons and the low-frequency phonons in proteins have been discussed in a re- 
cent paper [70].

As stated on the web-page of Vermont Photonics Technologies Corp. at Vermont

(http://www.sover.net/ bell/newFrontierpics.htm), "Study of low-frequency (or Terahertz frequency) motions in biomacromolecules holds a very exciting potential that could lead to revolutionize biophysics, molecular biology, and biomedicine."

For a systematic introduction of the low-frequency collective motion in biomacromolecules and its biological functions, refer to a comprehensive review article [71].

\subsection{Concluding Remarks on Mechanism}

Direct effects on electron (or hole) flow in DNA by the models cited are not proven. It is, however, easy to visualize how ELF-EMF could damage cellular processes and structures with the documented mechanisms described. A cell is an electrolyte rich, dipolar-proteinfilled water-dominated dielectric through which course many lipid membranes filled with voltage-gated and other types of channels designed for transmitting biochemical signals. It is easy to visualize how such a system could be affected by low frequency, strong timevarying magnetic fields and their associated electric fields, and so most attention has been focused on effects on electrolyte flow, protein dipole responses, and signal transduction at membranes. One interesting study used pulsed electric fields (not magnetic fields), a burst of high voltage, $40 \mathrm{kiloVolt} / \mathrm{cm}, 300$ nanosecond wide pulses to produce complete destruction of focal melanomas injected subcutaneously into mice [72]. Because electrodes must be placed on either side of the tumor there are severe practical restrictions on the applicability of such a technology to tumors in general, but such an approach can yield valuable information on the strength of the fields required to produce tumor destruction. The general conception has been that it is the electrical fields, not the magnetic fields per se that couple into biological structures to produce an effect. Consequently the pulsed electric field experiment can provide valuable information on the magnetic field parameters required to produce an effective electric field.

One must be cognizant, however, that the studies and models of Blank, Goodman, and Elson have raised the possibility that coupling of electromagnetic energy can occur as well directly through the magnetic fields. This possibility can be explained by the expressions $\mathrm{F}=\mathrm{qv} \mathrm{X}$ $B$ and curl $\mathrm{E}=\mathrm{dB} / \mathrm{dt}$ of classical electromagnetism, and remembering that a cell is filled with currents through membranes virtually at all times and possibly currents through DNA as well. The technology is available to fashion waveforms with far faster rise-times, shorter pulse-widths, far higher field strengths, varying repetition rates and burst modes than have been studied. In view of the fact that no adverse side-effects have been found and no abnormalities in normal tissue have been identified to date (possibly a very significant advantage over chemotherapy and conventional radiation) exciting opportunities are visible.

In view of these many interesting possibilities, and the promise communicated by the existing literature, a very strong case can and should be made for an investment into the potential of ELF-EMF in cancer therapeutics.

\section{EMF-DNA INTERACTION MECHANISMS: SIGNALING PATHWAYS}

The initial step in transmitting extracellular information from the plasma membrane to the nucleus of the cell is by NADH oxidase [73]. NADH then rapidly generates reactive oxygen species (ROS). These ROS stimulate matrix metalloproteinases which allows them to cleave and release heparin binding epidermal growth factor. This secreted factor actives the epidermal growth receptor which in turn activates the ERK cascade [73].

The major mechanism that regulates transcriptional activity in response to extracellular stimuli is the activation of the mitogen-activated protein kinase (MAPK) signaling cascades. There are three MAPK cascades that are implicated in exposures to ELF and RF. They are: 1) extracellular signal regulated kinase $1 \backslash 2$ (ERK), 2) c-Jun-terminal kinase (JNK), stress actrivated protein kinase (SAPK) and p38SAPK. Each of the cascades is composed of three to six tiers of protein kinases and their signals are transmitted by sequential phosphorylation and activation of the protein kinases in each of the tiers. Upon activation the protein kinases in various tiers phosphorylated and activated a large number of regulatory proteins which include a set of transcription factors, e.g., c-Jun, c-Fos, hsp27 and hsp70. Activation of the stress response is accompanied by activation of specific signal transduction cascades involved in regulating cell proliferation, differentiation and metabolism [74-77]. The MAPK pathways have been characterized in several cell types [74,78-81]. Exposure to nonthermal EMF as well as RF affects the expression of many cellular proteins [73-75].

The initial step in transmitting extracellular information from the plasma membrane to the nucleus of the cell is by NADH oxidase [73]. NADH then rapidly generates reactive oxygen species (ROS). These ROS stimulate matrix metalloproteinnases which allows them to cleave and release heparin binding epidermal growth factor. This secreted factor activates the epidermal growth receptor which in turn activates the ERK cascade [73].

Both EMF and RF activate the upregulation of the HSP70 gene and the induction of elevated levels of the hsp70 protein. This effect on RNA transcription and 
protein stability is controlled by specific protein transcription factors which are elements of the mitogen-activated-phospho-kinase (MAPK) cascade.

EMF also stimulate serum response factor which binds to the serum response element (SRE) through ERKmapk activation and is associated with injury and repair in in vivo and in vitro. The SRE site is on the promoter of an early response gene, c-fos, which under specific cellular circumstances has oncogenic properties. The c-fos promoter is EMF-sensitive; a 20 min exposure to $60 \mathrm{~Hz} 80 \mathrm{mG}$ sinusoidal fields significantly increased c-fos gene expression [82]. The SRE accessory protein, Elk-1, contains a growth-regulated transcriptional activation domain. ERK phosphorylation potentiates Elk-1 and transactivation at the c-fos SRE [8]. ELF-EMF exposure may also control protein regulation through the PI3-kinase pathway as inhibition results in an upregulation of collagen in response to ELF-EMF, suggesting an inhibitory role for PI3K in ELF-EMF induction. Furthermore, the role of nitric oxide/ cGMP signaling pathway has been implicated in pulsed EMF induced chondrocyte proliferation.

In studying human disorders, such as cancer, a stronger emphasis should be placed on model systems and noninvasive techniques for patient safety and ease of application for the treating physician. Information from the laboratory bench on non-human model organisms is under appreciated and very important.

\section{REFERENCES}

[1] M. H. Repacholi and B. Greenebaum, (1999) Interaction of static and extremely low frequency electric and magnetic fields with living systems: Health effects and research needs. Bioelectromagnetics. 20(3), 133-60.

[2] C. A. L. Bassett, (1995) Bioeletromagnetics in the service of medicine. Adv Chem, 250, 261-276.

[3] M. Blank and R. Goodman, (2004) Initial Interactions in Electromagnetic Field-Induced Biosynthesis. J. Cellular Physiology, 199, 359-363.

[4] M. Blank and R. A. Goodman, (2008) Mechanism for stimulation of biosynthesis by electromagnetic fields: Charge transfer in DNA and base pair separation. Journal of Cellular Physiology.

[5] H. Ito and C. A. Bassett, (1983) Effect of weak, pulsing electromagnetic fields on neural regeneration in the rat. Clin Orthop Relat Res, 181, 283-290.

[6] M. Jin, H. Lin, L. Han, M. Opler, S. Maurer, M. Blank, and R. Goodman, (1997) Biological and technical variables in myc expression in HL60 cells exposed to $60 \mathrm{~Hz}$ electromagnetic fields. Bioelectrochemistry and Bioenergetics, 44, 111-120.

[7] F. Shao, K. Augustyn, and J. K. Barton, (2005) Sequence dependence of charge transport through DNA domains. J Am Chem Soc, 127, 17445-17452.

[8] B. F. Sisken, M. Kanje, G. Lundborg, E. Herbst, and W. Kurtz, (1989) Stimulation o frat sciatic nerve regeneration with pulsed electromagnetic fields. Brain Res, 485,
309-316.

[9] B. F. Sisken, J. Walker, and M. Orgel, (1993) Prospects on clinical applications of electrical stimulation for nerve regeneration. J Cell Biochem, 51, 404-409.

[10] J. L. Walker, J. M. Evans, P. Meade, P. Resig, and B. F. Sisken, (1994) Gait-stance duration nas a measure of injury and recovery in the rat sciatic nerve model. J Neurosci Methods, 52, 47-52.

[11] J. M. Shallom, A. L. DiCarlo, D. Ko, L. M. Penafiel, and A. Nakai, (2002) Microwave exposure induces hsp70 and confers protection against hypoxia in chick embryos. J Cell Biochem, 86, 490-496.

[12] I. George, M. Geddis, Z. Lill, H. Lin, T. Gomez, M. Blank, M. Oz, and R. Goodman, (2008) Myocardial function improved by electromagnetic fields induction of stress protein hsp70. J Cellular Physiol, 216, 816-823. Published Online: DOI: 10.1002/jcp.21461.

[13] A. Albertini, B. Zucchini, G. Noera, R. Cadossi, C. P. Napoleone, and A. Pierangeli, (1999) Protective effect of low frequency low energy policy electromagnetic fields on acute experimental myocardial infarcts in rats. Bioelectromagnetics, 20, 372-377.

[14] A. Di Carlo, J. M. Farrell, and T. Litovitz, (1998) A simple experiment to study electromagnetic field effects: Protection induced by short-term exposures to $60 \mathrm{~Hz}$ magnetic fields. Bioelectromagnetics, 19, 498-500.

[15] R. Goodman and A. Henderson, (1988) Exposure of salivary gland cells to low frequency electromagnetic fields alters polypeptide synthesis. PNAS, 85, 3928- 3932.

[16] R. Goodman, C. A. L. Bassett, and A. Henderson, (1983) Pulsing electromagnetic fields induce cellular transcription. Science, 220, 1283-1285.

[17] R. Goodman, M. Blank, H. Lin, O. Khorkova, L. Soo, D.Weisbrot, and A. S. Henderson, (1994) Increased levels of hsp70 transcripts are induced when cells are exposed to low frequency electromagnetic fields. Bio- electrochem Bioenerg, 33, 115-120.

[18] R. Goodman and M. Blank, (1998) Magnetic field stress induces expression of hsp70. Cell Stress and Chaperones, 3, 79-88.

[19] H. Lin, M. Blank, K. Rossol-Haseroth, and R. Goodman, (2001) Regulating genes with electromagnetic response elements. J Cell Biochem, 81, 143-148.

[20] H. Lin, M. Head, M. Blank, L. Han, M. Jin, and R. Goodman, (1998) Myc-mediated transactivation of HSP70 expression following exposure to magnetic fields. J Cell Biochem, 69, 181-188.

[21] R. Palumbo, D. Capasso, F. Brescia, P. Mita, M. Sari, F. Bersani, and M. R. Scarfi, (2006) Effects on apoptosis and reactive oxygen species formation by Jurkat cells exposed to $50 \mathrm{~Hz}$ electromagnetic fields. Bioelec- tromagnetics, 27, 159-162.

[22] S. Tofani, D. Barone, M. Cintorino, M. M. de Santi, A. Ferrara, R. Orlassino, P. Ossola, F. Perogio, K. Rolfo, and F. Ronchetto, (2001) Static and ELF magnetic fields induce tumor growth inhibition and apoptosis. Bioelectromagnetics, 22, 419-428.

[23] E. Elson, (2009) The little explored efficacy of magnetic fields in cancer treatment, and postulation of the mechanism of action. Electromagnetic Biology and Medicine, in press. 
[24] E. D. Kirson, V. Dbalý, F. Tovaryš, J. Vymazal, J. F. Soustiel, A. Itzhaki, D. Mordechovich, S. S. Shirley, Z. Gurvich, R. chneiderman, Y. Wasserman, M. Salzberg, B. Ryffel, D. Goldsher, E. Dekel, and Y. Palti, (2007) Alternating electric fields arrest cell proliferation in animal tumor models and human brain tumors. PNAS, 104, 10152-10157.

[25] C. Polk and E. Postow, (1996) Eds. Handbook of Biological Effects of Electromagnetic Fields. 2nd Ed, CRC Press, Chapter 4.

[26] C. D. William, M. S. Markov, W. E. Hardman, and I. L. Cameron, (2001) Therapeutic electromagnetic field effects on angiogenesis and tumor growth. Anticancer Research, 21, 3887-3892.

[27] R. De Seze, S. Tuffet, J. M. Moreau, and B. Veyret, (2000) Effects of $100 \mathrm{mT}$ time varying margnetic fields on the growth of tumors in mice. Bioelectromagnetics, 21, 107-111.

[28] I. L. Cameron, L. Z. Sun, N. Short, W. E. Hardman, and C. D. Williams, (2005) Therapeutic electromagnetic field [TEMF] and gamma irradiation on human breast cancer xenograft growth, angiogenesis and metastasis. Cancer Cell International, $\mathbf{5 ( 2 3 )}$.

[29] D. Kultz, (2005) Molecular and evolutionary basis of the cellular stress response. Ann Rev Physiol, 67, 225-257.

[30] J. Topol, D. M. Ruden, and C. S. Parker, (1985) Sequences required for in vitro transcriptional activation of a drosophila hsp70 gene. Cell, 42, 527-537.

[31] M. T. Santini, A. Ferrante, G. Rainaldi, P. Indovina, P. L. Indovina, (2005) Extremely low frequency magnetic fields and apoptosis: A review. International Journal of Radiation Biology, 81, 1-11.

[32] A. R. Liboff, (1998) Electric-field ion cyclotron resonance. Bioelectromagnetics, 18, 85-87.

[33] V. V. Lednev, (1993) Possible mechanism for the effect of weak magnetic fields on biological systems: Correction of the basic expression and it consequences. Electricity and Magnetism in Biology and Medicine, Blank, M. Ed. San Francisco Press, San Francisco, 550- 552.

[34] S. Kwee, P. Raskmark, and S. Velizarov, (2001) Changes in cellular proeins due to environmental non-ionizing radiation. I. Heat-shock proteins. Electro- and Magnetobiology, 20, 141-152.

[35] M. Grattarola, A. Chiabrera, R. Vlvianl, and G. Parodl, (1985) Interactions between weak electromagnetic fields and biosystems: A summary of nine years of research, 4 (1), 211-226.

[36] L. Michael and S.G. Ernst, (2005) Applied AC and DC magnetic fields cause alterations in the mitotic cycle of early sea urchin embryos. 4(6), $231-240$.

[37] E. Elson, (2007) Developmental control in animals and a biological role for DNA charge transfer. Progress in Biophysics \& Molecular Biology, 95, 1-15.

[38] E. J. Merino, A. K. Boal, and J. K. Barton, (2008) Biological contexts for DNA charge transport chemistry. Current Opinion in Chemical Biology, 12, 229-237.

[39] C. Wan, T. Fiebig, S. O. Kelley, C. R. Treadway, and J. K. Barton, (1999) Femtosecond dynamics of DNA-mediated electron transfer. Proc Nat Acad Sci, USA, 96, 60146019.

[40] H. Lai and N. P. Singh, (2004) Magnetic-field-induced DNA strand breaks in brain cells of the rat. Environ- mental Health Perspectives, 112, 687-94.

[41] H. Lai and N. P. Singh, (1997) Acute exposure to a $60 \mathrm{~Hz}$ magnetic field increases DNA strand breaks in rat brain cells. Bioelectromagnetics, 18, 156-165.

[42] H. Lai and N. P. Singh, (2005) Interaction of microwaves and a temporally incoherent magnetic field on single and double DNA strand breaks in rat brain cells. Electromag Biol Med, 24, 23-29.

[43] G. Gordon, (2007) Designed electromagnetic pulsed therapy: Clinical applications. Journal of Cellular Physiology, 212, 579-582.

[44] G. Gordon, (2008) Extrinsic electromagnetic fields, low frequency (phonon) vibrations, and control of cell function: A non-linear resonance system. Journal of Biomedical Science and Engineering (JBiSE), 1, 152-156. (open accessible at http://www.srpublishing.org/journal/jbise/).

[45] K. C. Chou and N. Y. Chen, (1977) The biological functions of low-frequency phonons. Scientia Sinica, 20, 447457.

[46] C. Chothia and J. Janin, (1975) Principles of proteinprotein recognition. Nature, 256, 705-708.

[47] P. C. Painter and L. E. Mosher, (1979) The low-frequency Raman spectrum of an antibody molecule: Bovine IgG. Biopolymers, 18, 3121-3123.

[48] P. C. Painter, L. E. Mosher, and C. Rhoads, (1981) Lowfrequency modes in the Raman spectrum of DNA. Biopolymers, 20, 243-247.

[49] P. C. Painter, L. E. Mosher, and C. Rhoads, (1982) Lowfrequency modes in the Raman spectra of proteins. Biopolymers, 21, 1469-1472.

[50] H. Urabe and Y. Tominaga, (1982) Low-frequency collective modes of DNA double helix by Raman spectroscopy. Biopolymers, 21, 2477-2481.

[51] H. Urabe, Y. Tominaga, and K. Kubota, (1983) Experimental evidence of collective vibrations in DNA double helix Raman spectroscopy. Journal of Chemical Physics, 78, 5937-5939.

[52] H. Urabe, Y. Sugawara, M. Ataka, and A. Rupprecht, (1998) Low-frequency Raman spectra of lysozyme crystals and oriented DNA films: Dynamics of crystal water. Biophys J, 74, 1533-1540.

[53] P. Martel, (1992) Biophysical aspects of neutron scattering from vibrational modes of proteins. Prog Biophys Mol Biol, 57, 129-179.

[54] K. C. Chou, (1983) Low-frequency vibrations of helical structures in protein molecules. Biochemical Journal, 209, 573-580.

[55] K. C. Chou, (1983) Identification of low-frequency modes in protein molecules. Biochemical Journal, 215, 465-469.

[56] K. C. Chou, (1984) Low-frequency vibration of DNA molecules. Biochemical Journal, 221, 27-31.

[57] K. C. Chou, (1984) The biological functions of low-frequency phonons: 4. resonance effects and allosteric transition. Biophysical Chemistry, 20, 61-71.

[58] K. C. Chou, (1985) Prediction of a low-frequency mode in BPTI. International Journal of Biological Macromolecules, 7, 77-80.

[59] K. C. Chou, (1985) Low-frequency motions in protein molecules: Beta-sheet and beta-barrel. Biophysical Journal, 48, 289-297.

[60] K. C. Chou, (1989) Low-frequency resonance and co- 
operativity of hemoglobin. Trends in Biochemical Sciences, 14, 212.

[61] K. C. Chou, G. M. Maggiora, and B. Mao, (1989) Quasicontinuum models of twist-like and accordion-like lowfrequency motions in DNA. Biophysical Journal, 56, 295-305.

[62] K. C. Chou, (1986) Origin of low-frequency motion in biological macromolecules: A view of recent progress of quasi-continuity model. Biophysical Chemistry, 25, 105116.

[63] K. C. Chou, N. Y. Chen, and S. Forsen, (1981) The biological functions of low-frequency phonons: 2. Co-operative effects. Chemica Scripta, 18, 126-132.

[64] K. C. Chou, (1984) The biological functions of low-frequency phonons: 3 . Helical structures and micro-environment. Biophysical Journal, 45, 881-890.

[65] K. C. Chou, (1987) The biological functions of mechanism of allosteric transition in antibody molecules. Biopolymers, 26, 285-295.

[66] K. C. Chou and G. M. Maggiora, (1988) The biological functions of low-frequency phonons: 7. The impetus for DNA to accommodate intercalators. British Polymer Journal, 20, 143-148.

[67] K. C. Chou and B. Mao, (1988) Collective motion in DNA and its role in drug intercalation. Biopolymers, 27, 1795-1815.

[68] K. C. Chou, and Y. S. Kiang, (1985) The biological functions of low-frequency phonons: 5. A phenomenological theory. Biophysical Chemistry, 22, 219-235.

[69] K. C. Chou, C. T. Zhang, and G. M. Maggiora, (1994) Solitary wave dynamics as a mechanism for explaining the internal motion during microtubule growth. Biopolymers, 34, 143-153.

[70] Z. Sinkala, (2006) Soliton/exciton transport in proteins. J Theor Biol, 241, 919-927.

[71] K. C. Chou, (1988) Review: Low-frequency collective motion in biomacromolecules and its biological functions. Biophysical Chemistry, 30, 3-48.

[72] R. Nuccitelli, X. Chen, A. F. Pakhomov, W. H. Baldwin, S. Sheik, J. L. Pomicter, W. Ren, C. Osgood, R. J. Swanson, J. F. Kolb, S. J. Beebe, and K. H. Schoenbach, (2009) A new pulsed electric field therapy for melanoma disrupts the tumor's blood supply and causes complete remission without recurrence. Internal Journal of Cancer, 125, 438-445.

[73] J. Friedman, S. Kraus, Y. Hauptman, Y. Schiff, and R.
Seger, (2007) Mechanism of short-term ERK activation by electromagnetic fields at mobile phone frequencies. Biochem J., 405, 559-568.

[74] M. Jin, M. Blank, and R. Goodman, (2000) ERK1/2 Phosphorylation, Induced by Electromagnetic Fields, Diminishes During Neoplastic Transformation. Journal of Cellular Biochemistry, 78, 371-379.

[75] D. Leszczynski, S. Joenvaara, J. Reivinen, and R. Kuokka, (2002) Non-thermal activation of the hsp27/ p38MAPK stress pathway by mobile phone radiation in human endothelial cells: Molecular mechanism for cancer-and blood-brain barrier-related effects. Differentiation, 70, 120-129.

[76] M. Simko, (2004) Induction of cell activation processes by low frequency electromagnetic fields. Scientific World Journal, Suppl 2, 4, 4-22.

[77] M. Simko, C. Hartwig, M. Lantow, M. Lupke, M. O. Mattsson, Q. Rahman, and J. Rollwitz, (2006) Hsp70 expression and free radical release after exposure to non-thermal radio-frequency electromagnetic fields and ultrafine particles in human Mono Mac 6 cells. Toxicol Lett. 161, 73-82.

[78] R. Marais, J. Wynne, and R. Treisman, (1993) The SRF accessory protein Elk-1 contains a growth factor-regulated transcriptional activation domain. Cell, 73, 381393.

[79] R. Janecht, W. H. Ernst, V. Pigoud, and A. Nordheim, (1993) Activation of TCF Elk-1 by MAP Kinases. EMBO J., 12, 5097-5104.

[80] H. Gille, M. Kortenjann, O. Thomae, C. Moomaw, C. Slaughter, M. H. Cobb, and P. E. Shaw, (1995) ERK phosphorylation potentiates Elk-1-mediated ternary complex formation and transactivation. EMBO J., 14, 951-962.

[81] V. Sgambato, P. Vanhouttte, C. Pages, M. Rogard, L. Hipskind, M. J. Besson, and J. Caboche, (1998) In vivo expression and regulation of Elk-1, a target of the extracellular-regulated kinase signaling pathway, in the rat brain. J. Neurosci., 18, 214-226.

[82] S. Rao and A. S. Henderson, (1996) Regulation of c-fos is affected by electromagnetic fields. J Cell Biochem, 63, 358-365.

[83] X. Zhang, H. Zhang, C. Zheng, C. Li, X. Zhang, and W. Xiong, (2002) Cell Biology International, 26, 599-603.

[84] H. Lin, M. Blank, and R. Goodman, (1999) A magnetic field responsive domain in the human HSP70 promoter. J Cell Biochem., 75, 170-176. 


\section{Literature Cited}

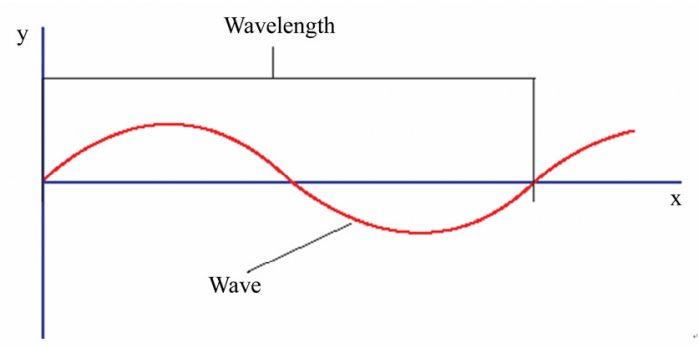

Legend: To facilitate an understanding of mechanisms, definition of the following terms should be helpful.

Wave - A wave is a disturbance traveling through a medium by which energy is transferred from one particle of the medium to another without causing any permanent displacement of the medium itself. The peaks of the wave are the maximum amount of energy.

Longitudinal Wave - a longitudinal wave is like a sound wave in air, where the pulse travels parallel to the direction of disturbance.

- Transverse Wave - a transverse wave is like a leaf floating in a lake. When a wave comes, the leaf goes up and then back down. So a transverse wave is where the motion is perpendicular to the direction of disturbance.
- Wavelength - A wavelength is the distance between any two repeating points, as shown in the diagram.

- $\quad$ Rise Time: The speed with which a pulse goes from zero to peak

- Measurement of field strength: Tesla/Gauss measure: 1 Tesla equals 10,000 gauss

- Frequency - The frequency of a wave is the number of times a point repeats in a certain amount of time.

While EMF signals come in various shapes (sine and square, pulsed etc.) through a sine wave or a series of sine waves. The delivery system of an electromagnetic field can be single pulse, repetive pulse and can be through Helmholtz and other coil configureation.

To understand the impact of EMF on cells and tissues it is important to understand specific acronyms. These include pulsed electromagnetic field (PEMF), time varying electromagnetic field (TVEMF) to pulsed electric stimulation (PES) and pulsed electromagnetic therapy (PEMT). The need to specifically design pulse and/or static field for maximal bio-efficacy. 